\title{
Transmission of malaria by intravenous drug misuse
}

\author{
Letter from Brazil
}

\author{
John Dunn
}

The invention of the hypodermic syringe in the 1850 s enabled drugs to be administered by the intravenous route. In a therapeutic setting this route is often preferred when rapid onset of action is required or if metabolism of the drug by the first-pass system is to be avoided. This rapidity of onset is a desirable effect which many illicit drug users seek, especially with drugs such as heroin, cocaine and amphetamines. As a drug user becomes more severely dependent, intravenous administration represents a more costeffective route compared with smoking, snorting or ingesting. If the needles and syringes are shared it also becomes the most efficient route for transmitting certain infectious diseases, as the epidemics of hepatitis B, hepatitis C and HIV among intravenous drug users (IVDUs) testify. Prevention of the spread of these diseases among IVDUs has become the cornerstone of treatment policies in the UK and many other countries.

In tropical countries the sharing of injecting equipment among IVDUs can lead to the spread of other blood-born infections, such as malaria. In Brazil only parts of the country are endemic for malaria, primarily the Amazonian regions, the states of Acre and Rondônia and rural areas of Marahão, Pará and Mato Grosso states which are situated in the north and central west regions of the country. In non-endemic areas, such as São Paulo in the south-east of Brazil, cases of malaria are almost always due to importation of the disease from endemic areas caused by human migration. São Paulo state is the most affluent state in Brazil and as a consequence is the recipient of a large amount of human migration from the poorer northern states.

In São Paulo malaria is a notifiable disease and any outbreaks of the disease are monitored and investigated by the Superintendência de Controle de Endemias (SUCEN). Since 1988 there have been several small outbreaks of malaria among IVDUs in the state of São Paulo. One of the best documented outbreaks occurred in 1990 in the city of Bauru (Barata et al., 1993), a city with a population of 260000 situated
$324 \mathrm{~km}$ from São Paulo city. Bauru is a city with an important rail interchange, with connections to various states but especially with the Amazonian region. During the first three weeks of July 1990 five cases of malaria were detected by local health services in Bauru. Autochthanous malaria had not occurred in the city since 1978. There followed an extensive epidemiological investigation which took place between 19 July and 13 September 1990.

This study revealed that none of the initial five patients had recently travelled to endemic areas, but all were using cocaine intravenously. Each of the patients was interviewed at home and asked whether and with whom they had shared injecting equipment in the last three months, whether they had received a blood transfusion in the last three months or travelled to an endemic area in the last 18 months. A blood sample was taken for confirmation of the presence of malarial parasites. In turn each of the contacts was visited and the process repeated until all contacts were accounted for.

One hundred and nineteen individuals were identified who had shared a needle and syringe with at least one of the people confirmed as suffering from malaria in the previous three months. One hundred and two individuals were interviewed ( 12 could not be located and five refused to be seen). Ninety-nine patients gave blood samples (three refused) from which 21 cases of malaria were identified. All patients were infected with Plasmodium vivax. The patients were treated with chloroquine and after the initial course treatment was continued on a weekly basis until the last patient had been contacted. Investigations revealed that one or more of the people involved in the outbreak had recently travelled to the Amazonian region and that this was how the disease was most probably introduced.

Transmission of malaria between IVDUs is not a new phenomenon and was described by Biggam in Egypt in 1929. There have been several outbreaks in the USA, each closely 
corresponding with the US's involvement in overseas conflicts. There were several documented outbreaks following the return to the US of servicemen involved in the Vietnam war. One of the greatest dangers from malaria to IVDUs is the spread of Plasmodium falciparum. In 193845 falciparum infections occurred among heroin users in New York City, nine of whom died. The diagnosis was delayed by the fact that the symptoms of malaria overlap with those of heroin withdrawal. The general population is not normally at risk from such outbreaks unless the natural vectors for the spread of malaria, the mosquito Anopheles freebornil and Anopheles punctipenis, are abundant in the local environment. Such an outbreak occurred in California in 1952 when 35 Campfire Girls contracted malaria due to $P$. vivax from an infected Korean War veteran by means of natural vectors.

Returning to the outbreak in Bauru in the state of São Paulo, in addition to examining blood specimens for malaria, researchers also tested them for syphilis, hepatitis B and HIV and found prevalences of $12 \%, 40 \%$ and $58 \%$ respectively. These figures are disturbingly high and reflect the degree of sharing which occurred among the users. Fifty-eight per cent of the sample admitted to having shared with between one and five people in the last three months and 20\% with between six and over 20 people. These figures are also of particular interest because of the nature of the sample. This was not a blased sample of drug users attending a treatment centre, but a sample that was obtained through contact tracing in a naturalistic setting in the community. Working from an initial group of five patients infected with malaria they traced a large network of 119 individuals who had shared injecting equipment with one another in the last three months.

This type of approach bears many similarities to the epidemiological technique of 'snowballing', which has been used in other community-based research studies of drug users, where the aim has been to obtain a more representative sample of a hidden population, namely drug users who are not in contact with treatment services. As a consequence, this study probably gives a more accurate figure for the prevalence of HIV among IVDUs than do other studies from the same state which have looked at samples of patients attending treatment centres (Mesquita et al., 1991). It also gives an indication of the huge amount of work that needs to be done in the areas of education, prevention and harm minimisation if these figures are not to rise further.

\section{References}

BARATA, L.C.B., ANDRIGUETT, M.T.M. \& DE MATOS, M.R. (1993) Surto de malárla induzida entre usúarias de drogas injetáveis. Revista Saúde Pública, 27, 9-14.

BIGGAM. A.G. (1929) Malignant malarla associated with the administration of heroin intravenously. Transactions of the Royal Soclety of Tropical Medictne and Hyglene, 23. 147-153.

MEsourta, F. et al (1991) Pllot study of HIV antibody seroprevalence among IVDUs in the city of Santos. São Paulo. Brazil. Abstracts of the Seventh International Conference on AIDS. Florence. (Abstract number: MC 3008).

John Dunn, CNPq Vistting Researcher, Departamento de Psiqiatria, Escola Paulista de Medicina. Rua Botucatu, 740, São Paulo-SP, Brazil, 04023 062 